\title{
Huge variation in obtaining ethical permission for a non-interventional observational study in Europe
}

\author{
Dylan W. de Lange ${ }^{1 *}$ D, Bertrand Guidet ${ }^{2,3,4}$, Finn H. Andersen ${ }^{5,6}$, Antonio Artigas ${ }^{7}$, Guidio Bertolini ${ }^{8}$, Rui Moreno ${ }^{9}$, \\ Steffen Christensen ${ }^{10}$, Maurizio Cecconi ${ }^{11}$, Christina Agvald-Ohmann ${ }^{12}$, Primoz Gradisek ${ }^{13}$, Christian Jung ${ }^{14}$, \\ Brian J. Marsh ${ }^{15}$, Sandra Oeyen ${ }^{16}$, Bernardo Bollen Pinto ${ }^{17}$, Wojciech Szczeklik ${ }^{18}$, Ximena Watson ${ }^{19}$, \\ Tilemachos Zafeiridis ${ }^{20}$ and Hans Flaatten ${ }^{21,22}$
}

\begin{abstract}
Background: Ethical approval (EA) must be obtained before medical research can start. We describe the differences in EA for an pseudonymous, non-interventional, observational European study.

Methods: Sixteen European national coordinators (NCs) of the international study on very old intensive care patients answered an online questionnaire concerning their experience getting EA.

Results: $N=8 / 16$ of the NCs could apply at one single national ethical committee (EC), while the others had to apply to various regional ECs and/or individual hospital institutional research boards (IRBs). The time between applying for EA and the first decision varied between 7 days and 300 days. In 9/16 informed consent from the patient was not deemed necessary; in 7/16 informed consent was required from the patient or relatives. The upload of coded data to a central database required additional information in 14/16. In 4/16 the NCs had to ask separate approval to keep a subject identification code list to de-pseudonymize the patients if questions would occur. Only 2/16 of the NCs agreed that informed consent was necessary for this observational study. Overall, 6/16 of the NCs were satisfied with the entire process and 8/16 were (very) unsatisfied. 11/16 would welcome a European central EC that would judge observational studies for all European countries.
\end{abstract}

Discussion: Variations in the process and prolonged time needed to get EA for observational studies hampers inclusion of patients in some European countries. This might have a negative influence on the external validity. Further harmonization of ethical approval process across Europe is welcomed for low-risk observational studies.

Conclusion: Getting ethical approval for low-risk, non-interventional, observational studies varies enormously across European countries.

\section{Background}

When doing medical research there is a potential disparity between the interests of the researcher and the patient who is going to be subjected to the medical research. For this reason, medical ethical approval has to be obtained before research can be started. The medical ethical committees (ECs) or institutional review boards (IRBs) should weight the benefits of medical research

\footnotetext{
* Correspondence: d.w.delange@umcutrecht.n!

${ }^{1}$ Department of Intensive Care Medicine, University Medical Center,

University Utrecht, Heidelberglaan 100, 3584, CX, Utrecht, The Netherlands

Full list of author information is available at the end of the article
}

against the potential harms for the patient. Interventional trials should only commence if, at least, the minimal legal, judicial and ethical standards are met. The European Union (EU) has issued a clinical trials directive (EU-CTD1) in 2001, which was implemented in the member states in 2004 [1]. Since then, more than 10 years have passed and a new clinical trials regulation has now been implemented (EU-CTD2) [2]. In this process, all countries have streamlined the process of getting approval for medical research and have appointed national medical ethical committees for the analyses and approval of medical research. This is especially well regulated for

(c) The Author(s). 2019 Open Access This article is distributed under the terms of the Creative Commons Attribution 4.0 International License (http://creativecommons.org/licenses/by/4.0/), which permits unrestricted use, distribution, and 
studies with an intervention, like (randomized) interventional trials.

However, observational research is generally considered as having a minimal risk for the participants. In many countries regulations have been developed to either exempt these studies from ethical review or provide the option of proportional review. These types of review are often delegated to the chair or smaller group of the ethical committee as they do not usually require a full board review [3]. However, up to date most of the research on timeliness of the ethical review has focused on those traditional interventional trials that require full review. We recently performed a multinational, observational study of very old critically ill patients (the VIP1study) [4]. The goal of the VIP1-study was to analyze whether frailty was associated with poor outcome in this elderly patient group. Performing this research provided us with an opportunity to explore how differently ethical approval processes are working in various countries. The VIP1-study is prototypical for low-risk observational research. In short, in this study we included 5021 patients over 80 years old from 311 ICUs. We looked at reasons for admission, severity of disease, frailty, treatments during ICU (e.g. mechanical ventilation, renal replacement therapy, and vasopressor treatment), limitations in life sustaining therapy and outcome at 30 days. The data was collected in central online database and apart from age, gender and study number no other data was collected that could lead to identification of the patients. National Coordinators (NCs) were to apply for national ethical approval at the appropriate EC. We aimed for a waiver of informed consent but various countries demanded written informed consent prior to inclusion. For a more detailed description of the methods and definitions we refer to the publication of the VIP1-study [4].

Here, we describe the differences in the processes to obtain ethical clearance in the participating European and EU affiliated countries and the opinions of the $\mathrm{Na}-$ tional Coordinators about the medical ethical approval process.

\section{Methods}

Originally, it was planned to get ethical approval from the participating countries in the first 6 months of 2016 . The first center in Europe to apply for ethical approval for the VIP1-study started in May 2016. The last country started with this procedure in October 2016. Each country had a designated National Coordinator (NC), who was responsible for the process of getting national ethical approval. In February 2017 an online questionnaire concerning the experience of getting ethical approval with 11 questions was sent out to 16 European NCs (Table 1).

\section{Definitions}

Every country has its own procedure to judge medical research protocols. We defined a "national medical ethical committee" as a committee that has the authority to judge an application for the entire country. A "regional medical ethical committee" has the authority to judge a medical research protocol for a particular region or state but not for the entire country. In many countries a local hospital ethical committee has to judge the medical research protocol as well. Their judgement can only be used as approval for that particular institute. We defined them as "institutional research boards" (IRBs).

\section{Ethical clearance}

The Regional Committee for Medical and Health Research Ethics, Western-Norway was asked to judge whether sending out a questionnaire on the ethical process to the NCs needed ethical committee review. They judged that sending out such a questionnaire was exempted from ethical review.

\section{Results}

Half of the countries $(n=8 / 16)$ needed apply to only one national medical ethical committee (national EC), while the others had to apply to the national ECs and subsequently to the individual hospital institutional research boards (IRBs) (question 1).

The most frequent body to be approached (question 2) was a local hospital institution review board (IRB) (67\%), followed by a regional EC (33\%) and a national EC (25\%) . In one country all participating hospitals had to send individual applications to their respective IRBs since there was no national medical ethical committee. Most hospitals had to provide more specific information regarding the upload of data (question 5) to a central database abroad $(n=14 / 16)$. One country had to apply for this at a separate EC, because the national EC was not allowed to judge data collection in a foreign country. In $n=4 / 16$ the NCs had to ask additional ethical approval to keep a local subject identification code list to depseudonymize the patients to be able to answer queries from the coordinating center (question 6). In 11 countries this was not necessary and in one country there was a separate application for this, resulting in two separate ethical approval rounds to the central EC.

The time from application until the first national approval (question 3) is shown in Fig. 1. The time-toapproval ranged from less than a week to more than 300 days.

In $n=9 / 16$ informed consent from the patient was not deemed necessary (question 4), and in $n=7 / 16$ informed consent was prospectively required from the patient or the relatives or retrospectively from the patient. In 5 countries consent from the patients was necessary in 
Table 1 The questionnaire to the national coordinators

\begin{tabular}{|c|c|c|}
\hline & Question & Answer possibilities \\
\hline Q1 & Did you have to apply to more than one body in order to get approval for the study? & $\begin{array}{l}\ldots \text { yes } \\
\ldots \text { no } \\
\ldots \text { other, please specify }\end{array}$ \\
\hline Q2 & If Yes on Q1: Which bodies (committees) did you apply for consent? & $\begin{array}{l}\ldots \text { a national committee/body } \\
\ldots \text { a regional committee/body } \\
\ldots \text { a local hospital intitutional research board } \\
\ldots \text { other, please specify }\end{array}$ \\
\hline Q3 & How long was the application process from start to decision (weeks)? & .... weeks \\
\hline Q4 & Did you have to get informed consent for this study? & $\begin{array}{l}\ldots \text { yes, from the patient } \\
\ldots \text { yes, from the family } \\
\ldots \text { yes, but in retrospect from the patient } \\
\ldots \text { No, not deemed necessary }\end{array}$ \\
\hline Q5 & $\begin{array}{l}\text { Is there a specific regulation when you send data electronically to a common } \\
\text { database in another country? }\end{array}$ & $\begin{array}{l}\text {... no } \\
\ldots \text { yes, it must be specified in detail on the application } \\
\ldots \text { yes, there is a seperate body and an independent } \\
\text { application }\end{array}$ \\
\hline Q6 & $\begin{array}{l}\text { Did you have to seek allowance to have your own (local) file/database with names } \\
\text { of the included patients from your unit? }\end{array}$ & $\begin{array}{l}\ldots \text { yes } \\
\ldots \text { no } \\
\ldots \text { never thought of it }\end{array}$ \\
\hline Q7 & If YES from patient or family on Q4, how often was consent denied? & $\begin{array}{l}\ldots \text { never } \\
\ldots \text { occasionally } \\
\ldots \text { sometimes } \\
\ldots \text { frequently }\end{array}$ \\
\hline Q8 & $\begin{array}{l}\text { Do you consider (personal opinion) it necessary to have informed consent for such a } \\
\text { study? }\end{array}$ & $\begin{array}{l}\ldots \text { yes } \\
\ldots \text { no } \\
\ldots \text { uncertain } \\
\ldots \text { other, please specify }\end{array}$ \\
\hline Q9 & All in all: How satisfied are you with the ethical clearance process for this study? & $\begin{array}{l}\ldots \text { very satisfied } \\
\ldots \text { satisfied } \\
\ldots \text { neutral } \\
\ldots \text { unsatisfied } \\
\ldots \text { very unsatisfied } \\
\ldots \text { other, please specify }\end{array}$ \\
\hline Q10 & $\begin{array}{l}\text { What is your opinion of having a common EU regulation on research ethics, and that } \\
\text { acceptance in one country will bind the rest (similar to drug-approval regulations) }\end{array}$ & $\begin{array}{l}\ldots \text { I am in favour of this } \\
\ldots \text { I am not sure } \\
\ldots \text { I am against this } \\
\ldots \text { Other, please specify }\end{array}$ \\
\hline Q11 & The name of your country & $\ldots$ \\
\hline
\end{tabular}

advance, and in one country survivors had to consent in retrospect and only then was it allowed to use their data. One country allowed for consent by the family (proxy) without confronting the patient. Only $n=2 / 16$ of the NCs agreed that informed consent was necessary for such an observational study (question 8).

Overall, $n=6 / 16$ of the NCs were satisfied with the entire process of ethical approval, $n=8 / 16$ were (very) unsatisfied with the process and $n=2 / 16$ were neutral (time consuming but necessary) (question 9)(Fig. 2).

However, there was no clear correlation between the time-to-approval and being dissatisfied $(p=0.622) . N=$ $11 / 16$ would welcome a European central EC that would judge observational studies for all European countries, while $n=5 / 16$ were "not sure" fearing "more bureaucracy" in the ethical approval process (question 10).

\section{Discussion}

International observational studies of consecutive patients can reveal important differences in admission policies, treatments and outcomes of certain subgroups of ICU patients. Such observational studies often collect pseudo-anonimized or coded patient information and no particular treatment or intervention is imposed upon the subjects of the study. The potential harm to the subjects is, therefore, negligible and most would claim none. One could assume that these observational studies are assessed by the national ethical committees in an identical swift modus and the need for informed consent is waived. On the contrary, here we describe that this process went from smooth to such a prolonged period that one country could not even enter the study before the proposed end date [5]. 


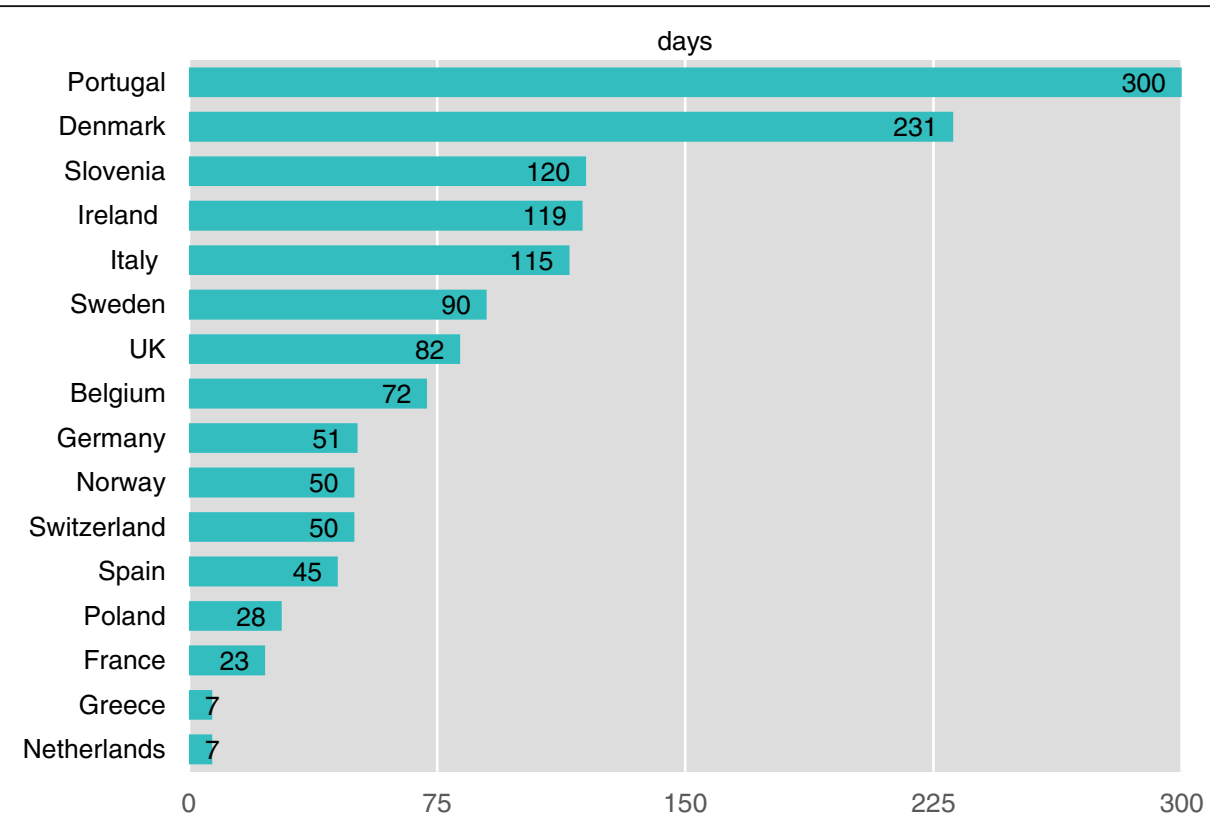

Fig. 1 Time between application and first approval. Time between application and first approval to start or rejection from national Ethical Committee(s) or Institutional Research Board(s)

In this survey, covering 16 European countries, we have demonstrated that there is very large variety of the ethical processes and outcome from either national ECs', regional ECs' or IRBs' approval with regard to an identical study protocol. In most countries, more than one level of ethical approval had to be approached. Often a national EC needed to assess the research protocol and, once approved, the local IRBs needed to consent with the protocol as well. The time from applications to decision was unexpectedly large and only a few countries received feedback within a month from their national EC [6-8]. After a national EC has cleared the study local IRBs need to assess whether the study can be performed at their institution. Sometimes local IRBs are stricter than their national ECs and sometimes these IRBs needed additional information that was lacking from the original study protocol. Clearly, the differences in "approval time" are not solely the responsibility of the ethical committees. Sometimes insufficient information in the protocol is reason for additional queries. This will lead to additional delay and the majority of ICUs needed additional time between start of the national EC procedure and the final local IRB approval [9]. Moreover, after this study was performed most of the European countries that have participated in our research have implemented the new General Data Protection Regulation (GDPR) which has had a major impact on the collection of observational data [10].

Another major disparity between countries was the need for informed consent. A basic requisite for medical research is that every patient has the right to decide whether or not he/she will participate in medical research. However, if the patient is unable to consent because of severe illness informed consent could be asked

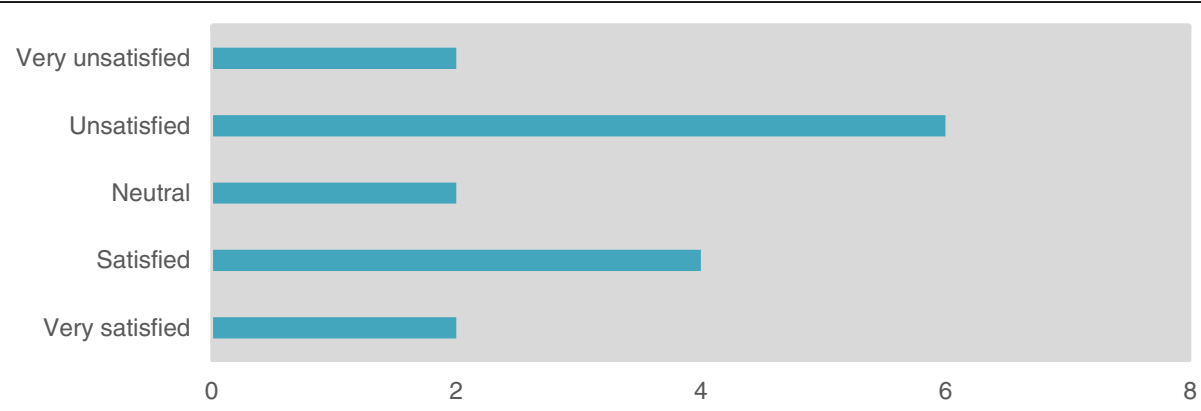

Fig. 2 Overall satisfaction with the ethical approval rate. There was no obvious relation between being satisfied/very satisfied and the length of the approval process or a concordance between satisfaction and wanting centralized EU approval 
retrospectively. However, some patients might not be able to give informed consent afterwards (because they are deceased or cognitively impaired). Leaving these patients out of the analyses might hamper the external validation of the research. In such cases the researchers can apply for a waiver of informed consent to the EC. The EC can grant such a waiver provided that the research poses important societal value and the research poses no more than minimal risk to the participants. We think this applies to observational studies in critically ill, elderly patients. The majority of countries were allowed to perform this study without any informed consent from the patient, but in a substantial amount of countries such permission had to be sought before inclusion of the patient [11]. If a patient is, for medical reasons, not able to consent (e.g. unconscious, sedated, etc.) and subsequently dies, this will create potential loss in patient recruitment subsequently leading to a selection bias and this may diminish the validity of the study [12]. A potential solution is to accept that survivors may claim their data to be removed (retrospective consent) but allow inclusion of data from deceased patients, despite the bias that also this option can introduce on the final results $[13,14]$. However, only one country used this method and from the 226 included patients only one patient opted for removal of his data. The majority of national coordinators $(n=14 / 16)$ judged this observational study as having such a low potential of harm to the subjects that they would not need informed consent for this study. However, this leaves $n=2 / 16$ that are convinced that consent is necessary even for anonymous observational registries.

Half of the national coordinators were not satisfied with the process. There was no obvious concordance between the time needed to get the study approved by the national EC and satisfaction. However, the countries in which the verdict was received swiftly were invariably satisfied with the entire process. Clearly a swift approval process leads to higher satisfaction. Especially in countries with a very prolonged EC process the national coordinators were dissatisfied and more willing to accept a centralized European ethical clearance entity for noninterventional studies/registries. At present, a new clinical trials directive (EU-CTD2) is being implemented. However, this directive particularly focuses on interventional trials (that, indeed, have a high potential risk of harm for the medical subjects). This leaves a gap in international legislation for pseudo-anonymous observational registries. The majority of the national coordinators would welcome a central European ethical organization for observational studies which would speed up national and local approvals. The general consensus was that such a non-interventional study should not take prolonged periods to be approved.
We have learned that we need to allow for up to one year to get all the countries to approve such a study [15, 16]. Not incorporating this "waiting time" in study protocols will lead to loss of countries and will, therefore, diminish the external validity of the findings for the European population. We presume more delay in approval for studies with a higher potential of harm for patients (like interventional studies or even patient questionnaires) $[17,18]$.

Obviously, this analysis has limitations. We only asked the national coordinators of the VIP1-study to provide us with their ethical approval experiences. After the national coordinators had gained ethical clearance many local researchers needed to go through a similar experience at their local IRBs. A swift approval at a national level may not be followed by a similarly swift approval at a local level, or vice versa. Such data were not incorporated in this analysis. Obviously, the results from our study are not necessarily transferrable to other studies (limited external validity).

\section{Conclusion}

We conclude that further harmonization between European countries in ethical clearance for observational, non-interventional studies and registries is desirable. However, these differences between European countries need to be considered and incorporated in the research plan in order to prevent missing important contributors.

\section{Acknowledgements}

This study was supported in kind (but not financially) by the European Society of Intensive Care Medicine (ESICM).

\section{Authors' contributions}

DL has made substantial contributions to the conception and design of the work; has aided in the acquisition, analysis and interpretation of data and has drafted the work and substantively revised it. He also has approved the submitted version (and any substantially modified version that involves the author's contribution to the study) and has agreed both to be personally accountable for the author's own contributions and to ensure that questions related to the accuracy or integrity of any part of the work, even ones in which the author was not personally involved, are appropriately investigated, resolved, and the resolution documented in the literature. BG has made substantial contributions to the conception and design of the work; has aided in the acquisition, analysis and interpretation of data and has drafted the work and substantively revised it. He also has approved the submitted version and has agreed both to be personally accountable for the author's own contributions and to ensure that questions related to the accuracy or integrity of any part of the work, even ones in which the author was not personally involved, are appropriately investigated, resolved, and the resolution documented in the literature. FA has aided in the acquisition and has drafted the work and substantively revised it. He also has approved the submitted version and has agreed both to be personally accountable for the author's own contributions and to ensure that questions related to the accuracy or integrity of any part of the work, even ones in which the author was not personally involved, are appropriately investigated, resolved, and the resolution documented in the literature. AA has aided in the acquisition and has drafted the work and substantively revised it. He also has approved the submitted version and has agreed both to be personally accountable for the author's own contributions and to ensure that questions related to the accuracy or integrity of any part of the work, even ones in which the author was not personally involved, are appropriately investigated, resolved, and the 
resolution documented in the literature. GB has aided in the acquisition and has drafted the work and substantively revised it. He also has approved the submitted version and has agreed both to be personally accountable for the author's own contributions and to ensure that questions related to the accuracy or integrity of any part of the work, even ones in which the author was not personally involved, are appropriately investigated, resolved, and the resolution documented in the literature. RM has aided in the acquisition and has drafted the work and substantively revised it. He also has approved the submitted version and has agreed both to be personally accountable for the author's own contributions and to ensure that questions related to the accuracy or integrity of any part of the work, even ones in which the author was not personally involved, are appropriately investigated, resolved, and the resolution documented in the literature. SC has aided in the acquisition and has drafted the work and substantively revised it. He also has approved the submitted version and has agreed both to be personally accountable for the author's own contributions and to ensure that questions related to the accuracy or integrity of any part of the work, even ones in which the author was not personally involved, are appropriately investigated, resolved, and the resolution documented in the literature. MC has aided in the acquisition and has drafted the work and substantively revised it. He also has approved the submitted version and has agreed both to be personally accountable for the author's own contributions and to ensure that questions related to the accuracy or integrity of any part of the work, even ones in which the author was not personally involved, are appropriately investigated, resolved, and the resolution documented in the literature. CAO has aided in the acquisition and has drafted the work and substantively revised it. He also has approved the submitted version and has agreed both to be personally accountable for the author's own contributions and to ensure that questions related to the accuracy or integrity of any part of the work, even ones in which the author was not personally involved, are appropriately investigated, resolved, and the resolution documented in the literature. PG has aided in the acquisition and has drafted the work and substantively revised it. He also has approved the submitted version and has agreed both to be personally accountable for the author's own contributions and to ensure that questions related to the accuracy or integrity of any part of the work, even ones in which the author was not personally involved, are appropriately investigated, resolved, and the resolution documented in the literature. CJ has aided in the acquisition and has drafted the work and substantively revised it. He also has approved the submitted version and has agreed both to be personally accountable for the author's own contributions and to ensure that questions related to the accuracy or integrity of any part of the work, even ones in which the author was not personally involved, are appropriately investigated, resolved, and the resolution documented in the literature. BM has aided in the acquisition and has drafted the work and substantively revised it. He also has approved the submitted version and has agreed both to be personally accountable for the author's own contributions and to ensure that questions related to the accuracy or integrity of any part of the work, even ones in which the author was not personally involved, are appropriately investigated, resolved, and the resolution documented in the literature. SO has aided in the acquisition and has drafted the work and substantively revised it. He also has approved the submitted version and has agreed both to be personally accountable for the author's own contributions and to ensure that questions related to the accuracy or integrity of any part of the work, even ones in which the author was not personally involved, are appropriately investigated, resolved, and the resolution documented in the literature. BB has aided in the acquisition and has drafted the work and substantively revised it. He also has approved the submitted version and has agreed both to be personally accountable for the author's own contributions and to ensure that questions related to the accuracy or integrity of any part of the work, even ones in which the author was not personally involved, are appropriately investigated, resolved, and the resolution documented in the literature. WS has aided in the acquisition and has drafted the work and substantively revised it. He also has approved the submitted version and has agreed both to be personally accountable for the author's own contributions and to ensure that questions related to the accuracy or integrity of any part of the work, even ones in which the author was not personally involved, are appropriately investigated, resolved, and the resolution documented in the literature. XW has aided in the acquisition and has drafted the work and substantively revised it. He also has approved the submitted version and has agreed both to be personally accountable for the author's own contributions and to ensure that questions related to the accuracy or integrity of any part of the work, even ones in which the author was not personally involved, are appropriately investigated, resolved, and the resolution documented in the literature. TZ has aided in the acquisition and has drafted the work and substantively revised it. He also has approved the submitted version and has agreed both to be personally accountable for the author's own contributions and to ensure that questions related to the accuracy or integrity of any part of the work, even ones in which the author was not personally involved, are appropriately investigated, resolved, and the resolution documented in the literature. HF has made substantial contributions to the conception and design of the work; has aided in the acquisition, analysis and interpretation of data and has drafted the work and substantively revised it. He also has approved the submitted version and has agreed both to be personally accountable for the author's own contributions and to ensure that questions related to the accuracy or integrity of any part of the work, even ones in which the author was not personally involved, are appropriately investigated, resolved, and the resolution documented in the literature.

\section{Funding}

This study did not receive any funding. Not applicable.

\section{Availability of data and materials}

The datasets used and/or analyzed during the current study are available from the corresponding author on reasonable request.

\section{Ethics approval and consent to participate}

Before publication of the data we asked the Regional Committee for Medical and Health Research Ethics, Western-Norway whether asking the national coordinators their experiences and using these data for a scientific publications needed ethical approval. The Regional Committee for Medical and Health Research Ethics, Western-Norway exempted sending out the questionnaire and using the information for a scientific publications from ethical review (30.08.2017).

\section{Consent for publication}

Not applicable.

\section{Competing interests}

The authors do not have competing interests concerning this study.

\section{Author details}

${ }^{1}$ Department of Intensive Care Medicine, University Medical Center, University Utrecht, Heidelberglaan 100, 3584, CX, Utrecht, The Netherlands. ${ }^{2}$ Hôpitaux de Paris, Hôpital Saint-Antoine, Service de Réanimation Médicale, 75012 Paris, France. ${ }^{3}$ Sorbonne Universités, UPMC Univ Paris 06, UMR_S 1136, Institut Pierre Louis d'Epidémiologie et de Santé Publique, 75013 Paris, France. ${ }^{4}$ INSERM, UMR_S 1136, Institut Pierre Louis d'Epidémiologie et de Santé Publique, Paris, France. ${ }^{5}$ Department of Anesthesia and Intensive Care, Møre and Romsdal Health Trust, Ålesund Hospital, Ålesund, Norway. ${ }^{6}$ Department of Circulation and Imaging, Faculty of Medicine, NTNU, Norwegian University of Science and Technology, Trondheim, Norway. ${ }^{7}$ Department of Intensive Care Medicine, CIBER Enfermedades Respiratorias, Corporacion Sanitaria Universitaria Parc Tauli, Autonomous University of Barcelona, Sabadell, Spain. ${ }^{8}$ Laboratory of Clinical Epidemiology, GiViTI Coordinating Center, department of Public Health, IRCCS - "Mario Negri" Institute for Pharmacological Research, Ranica (Bergamo), Italy. ${ }^{9}$ Unidade de Cuidados Intensivos Neurocríticos, Hospital de São José, Centro Hospitalar de Lisboa Central, Lisbon, Portugal. ${ }^{10}$ Department of Anaesthesia and Intensive Care Medicine, Aarhus University Hospital, Aarhus, Denmark. ${ }^{11}$ Department Anaesthesia and Intensive Care Units, IRCCS Istituto Clinico Humanitas, Humanitas University, Milan, Italy. ${ }^{12}$ Department of Anaesthesiology and Intensive Care, Department of Clinical Intervention and Technology, Karolinska University Hospital, Huddinge, Sweden. ${ }^{13}$ Clinical Department of Anaesthesiology and Intensive Therapy, University Medical Centre Ljubljana, Ljubljana, Slovenia. ${ }^{14}$ Division of Cardiology, Pulmonology and Vascular Medicine, University Hospital Düsseldorf, Heinrich-Heine-University, Düsseldorf, Germany. ${ }^{15}$ Department of Surgery, Mater Misericordiae University Hospital, Dublin, Ireland. ${ }^{16}$ Department of Intensive Care Medicine, Ghent University Hospital, Ghent, Belgium. ${ }^{17}$ Division of Anaesthesiology, Department of Anaesthesiology, Clinical Pharmacology and Intensive Care (APSI), Geneva University Hospitals, Geneva, Switzerland. ${ }^{18}$ Division of Intensive Care and Perioperative Medicine, 2nd Department of Medicine, 
Jagiellonian University Medical College, Krakow, Poland. ${ }^{19}$ Intensive Care, Kingston Hospital, Kingston, UK. ${ }^{20}$ Intensive Care Unit, General Hospital of Larissa, Larissa, Greece. ${ }^{21}$ Department of Anaesthesia, Haukeland University Hospital, University of Bergen, Bergen, Norway. ${ }^{22}$ Intensive Care and Department of Clinical Medicine, Haukeland University Hospital, University of Bergen, Bergen, Norway.

Received: 28 June 2018 Accepted: 17 May 2019

Published online: 03 June 2019

\section{References}

1. Singer EA, Mullerner M. Implications of the EU directive on clinical trials for emergency medicine. BMJ. 2002;324:1169-70. https://doi.org/10.1136/bmj. 324.7347.1169

2. Giannuzzi V, Altavilla A, Ruggieri L, et al. Clinical trial application in Europe: what will change with the new regulation? Sci Eng Ethics. 2016;22(2):45166. https://doi.org/10.1007/s11948-015-9662-0.

3. Perlman M. Ethical issues in 'observational research. Paediatr Child Health. 2000 Mar;5(2):89-90. https://doi.org/10.1093/pch/5.2.89.

4. Flaatten $H$, De Lange DW, Morandi A, Andersen FH, Artigas A, Bertolini G, Boumendil A, Cecconi M, Christensen S, Faraldi L, Fjølner J, Jung C, Marsh B, Moreno R, Oeyen S, Öhman CA, Pinto BB, Soliman IW, Szczeklik W, Valentin A, Watson X, Zaferidis T, Guidet B. VIP1 study group. The impact of frailty on ICU and 30-day mortality and the level of care in very elderly patients ( $\geq 80$ years). Intensive Care Med. 2017;43(12):1820-8. https://doi.org/10.1007/ s00134-017-4940-8.

5. Hunter D. Is research ethics regulation really killing people? Med J Austr. 2015;202(6):338-9. https://doi.org/10.5694/mja14.00338.

6. Al-Shahi Salman R, Beller E, Kagan J et al. Increasing value and reducing waste in biomedical research regulation and management. Lancet 2014;11; 383(9912):176-185. doi: https://doi.org/10.1016/S0140-6736(13)62297-7.

7. Goodyear-Smith F, Lobb B, Davies G, et al. International variation in ethics committee requirements: comparisons across five westernised nations. BMC Med Ethics. 2002;19(3):E2.

8. Kearney A, McKay A, Hickey $H$, et al. Opening research sites in multicentre clinical trials within the UK: a detailed analysis of delays. BMJ Open 2014;16; 4(9):e005874. doi: https://doi.org/10.1136/bmjopen-2014-005874.

9. Sandy J, Kilpatrick N, Persson M, et al. Why are multi-centre clinical observational studies still so difficult to run? Br Dent J. 2011;211(2):59-61. https://doi.org/10.1038/sj.bdj.2011.570.

10. Décret $n^{\circ}$ 2016-1537 du 16 novembre 2016 relatif aux recherches impliquant la personne humaine JORF n0267 du 17 novembre 2016. https://www.legifrance.gouv.fr/eli/decret/2016/11/16/AFSP1621392D/jo/ texte. Website last accessed: 25th May 2019.

11. Pollock K. Procedure versus process: ethical paradigms and the conduct of qualitative research. BMC Med Ethics. 2012;13:25. https://doi.org/10.1186/ 1472-6939-13-25.

12. Kompanje EJ, Maas Al, Menon DK, et al. Medical research in emergency research in the European Union member states: tensions between theory and practice. Intensive Care Med. 2014;40(4):496-503. https://doi.org/10. 1007/s00134-014-3243-6.

13. Lemaire F, Blanch L, Cohen SL, et al. Informed consent for research purposes in intensive care patients in Europe--part II. An official statement of the European Society of Intensive Care Medicine. Working Group on Ethics Intensive Care Med 1997;23(4):435-439. PubMed PMID: 9142584.

14. Jansen TC, Kompanje EJO, Druml C, et al. Deferred consent in emergency intensive care research: what if the patient dies early? Use the data or not? Intensive Care Med. 2007;33(5):894-900. https://doi.org/10.1007/s00134-0070580-8.

15. de Lange DW, Guidet B, Moreno R, Christensen S, Flaatten F. On behalf of the VIP1 study group. Huge variation in obtaining ethical permission for a non-intervention observational study in Europe. Intensive Care Medicine Experimental. 2017;5(Suppl 2):0370.

16. De Smit E, Kearns LS, Clarke L, et al. Heterogeneity of human research ethics committees and research governance offices across Australia: an observational study. Australas Med J. 2016;9(2):33-9. https://doi.org/10.4066/ AMJ.2015.2587.
17. Ahmed AH, Nicholson KG. Delays and diversity in the practice of local research ethics committees. J Med Ethics. 1996;22:263-6. https://doi.org/10. 1136/jme.22.5.263.

18. Barnett A, Campbell MJ, Shield C, et al. The high costs of getting ethical and site-specific approvals for multi-Centre research. Research Integrity and peer review. 2016;1:16. https://doi.org/10.1186/s41073-016-0023-6.

\section{Publisher's Note}

Springer Nature remains neutral with regard to jurisdictional claims in published maps and institutional affiliations.
Ready to submit your research? Choose BMC and benefit from:

- fast, convenient online submission

- thorough peer review by experienced researchers in your field

- rapid publication on acceptance

- support for research data, including large and complex data types

- gold Open Access which fosters wider collaboration and increased citations

- maximum visibility for your research: over $100 \mathrm{M}$ website views per year

At BMC, research is always in progress.

Learn more biomedcentral.com/submissions 